\title{
Loss of Swiss Cheese/Neuropathy Target Esterase Activity Causes Disruption of Phosphatidylcholine Homeostasis and Neuronal and Glial Death in Adult Drosophila
}

\author{
Max Mühlig-Versen, ${ }^{2}$ Alexandre Bettencourt da Cruz, ${ }^{1}$ Jakob-Andreas Tschäpe, ${ }^{1}$ Markus Moser, ${ }^{3}$ Reinhard Büttner, ${ }^{4}$ \\ Karin Athenstaedt, ${ }^{5}$ Paul Glynn, ${ }^{6}$ and Doris Kretzschmar ${ }^{1,2}$ \\ ${ }^{1}$ Center for Research on Occupational and Environmental Toxicology, Oregon Health and Sciences University, Portland, Oregon 97239, ${ }^{2}$ Lehrstuhl für \\ Entwicklungsbiologie, Universität Regensburg, 93053 Regensburg, Germany, ${ }^{3}$ Max-Planck-Institut of Biochemistry, Department of Molecular Medicine, \\ 82152 Martinsried, Germany, ${ }^{4}$ Institut für Pathologie, Universität Bonn, 53127 Bonn, Germany, ${ }^{5}$ Institut für Biochemie und Lebensmittelchemie, \\ Technische Universität Graz, 8010 Graz, Austria, and ${ }^{6}$ Medical Research Council Toxicology Unit, University of Leicester, Leicester LE1 9HN, United \\ Kingdom
}

The Drosophila Swiss cheese (sws) mutant is characterized by progressive degeneration of the adult nervous system, glial hyperwrapping, and neuronal apoptosis. The Swiss cheese protein (SWS) shares 39\% sequence identity with human neuropathy target esterase (NTE), and a brain-specific deletion of SWS/NTE in mice causes a similar pattern of progressive neuronal degeneration. NTE reacts with organophosphate compounds that cause a paralyzing axonal degeneration in humans and has been shown to degrade endoplasmic reticulum-associated phosphatidylcholine (PtdCho) in cultured mammalian cells. However, its function within the nervous system has remained unknown. Here, we show that both the fly and mouse SWS proteins can rescue the defects that arise in sws mutant flies, whereas a point mutation in the proposed active site cannot restore SWS function. Overexpression of catalytically active SWS caused formation of abnormal intracellular membraneous structures and cell death. Cell-specific expression revealed that not only neurons but also glia depend autonomously on SWS. In wild-type flies, endogenous SWS was detected by immmunohistochemistry in the endoplasmic reticulum (the primary site of PtdCho processing) of neurons and in some glia. sws mutant flies lacked NTE-like esterase activity and had increased levels of PtdCho. Conversely, overexpression of SWS resulted in increased esterase activity and reduced PtdCho. We conclude that SWS is essential for membrane lipid homeostasis and cell survival in both neurons and glia of the adult Drosophila brain and that NTE may play an analogous role in vertebrates.

Key words: Drosophila; NTE; neurodegeneration; organophosphate-induced delayed neuropathy; phospholipids; ER

\section{Introduction}

Age-dependent neurodegeneration in the adult nervous system is often characterized by a spectrum of abnormalities including neuronal and glial apoptosis, reactive gliosis or abnormal glial sprouting, and vacuolar lesions. We have characterized a number of Drosophila mutants that exhibit several of these degenerative phenotypes. In particular, the Swiss cheese (sws) mutant shows age-dependent neurodegeneration detectable by the formation of spongiform lesions within the CNS, accompanied by neuronal apoptosis (Kretzschmar et al., 1997). In addition, glial cells in these animals form multilayered wrappings around neurons. In

\footnotetext{
Received July 1, 2004; revised Feb. 2, 2005; accepted Feb. 3, 2005.

This work was supported by grants to D.K. from the Deutsche Forschungsgemeinschaft (Forschergruppe BU672/ 5-1) and the National Institutes of Health (NS047663-01) and by grants to M.M. from the Deutsche Forschungsgemeinschaft (Mo 927/1-1, 1-2). We thank Tina Wanke, Uschi Roth, and Laura Reese for technical assistance and Sinje Maruhn for her support of the injection crew.

Correspondence should be addressed to Doris Kretzschmar, Center for Research on Occupational and Environmental Toxicology L606, 3181 Southwest Sam Jackson Park Road, Oregon Health and Sciences University, Portland OR 97201-3098. E-mail: kretzsch@ohsu.edu.

DOI:10.1523/JNEUROSCI.5097-04.2005

Copyright $\odot 2005$ Society for Neuroscience $\quad$ 0270-6474/05/252865-09\$15.00/0
}

late wild-type pupae, glial cells form single-layered sheaths around neuronal cell bodies and axons. In sws mutant pupae, multilayered glial wrappings appear, which eventually form large whorls around degenerating neurons in adults.

The SWS protein shares homology with open reading frames in yeast, nematodes, and with vertebrate neuropathy target esterase (NTE) (Lush et al., 1998). NTE is the molecular target of those organophosphorus compounds that induce delayed neuropathy (OPIDN) in vertebrates (Johnson, 1990; Lotti, 1992; Atkins and Glynn, 2000; Glynn, 2000; Moretto, 2000). This neuropathy is characterized by swelling and degeneration of long axons in the CNS and peripheral nervous system (Dyer et al., 1991, 1992). OPIDN was first brought to public attention by a poisoning epidemic in the United States in the 1920s (Smith et al., 1930); however, the mechanisms leading to this syndrome and the function of NTE are still unknown.

In vitro, NTE is detected by its organophosphate (OP)sensitive serine esterase activity, assayed by the hydrolysis of phenyl valerate (Johnson, 1977). This assay provided a convenient means of quantifying NTE activity and measuring the effects of neuropathic OPs, but it did not provide insight into the endoge- 
nous substrate(s). Recently, in mammalian cell lines, NTE has been shown to degrade endoplasmic reticulum (ER)-associated phosphatidylcholine (PtdCho) and has been proposed to play a role in membrane lipid homeostasis (Zaccheo et al., 2004). However, it remains to be determined whether PtdCho or other membrane lipids comprise substrates for NTE in vivo.

Disruption of NTE/SWS in mice caused embryonic lethality with defects in the formation of the labyrinth layer, survival of secondary giant cells, and blood vessel formation (Winrow et al., 2003; Moser et al., 2004).

Notably, brain-specific deletions of NTE in mice caused phenotypes strikingly similar to sws (Akassoglou et al., 2004). After 6 weeks of age, hippocampal and thalamic neurons displayed vacuolation, abnormal membranous folds, and myelin figures, whereas Purkinje cells underwent cell death. The remarkable similarity of the neurodegenerative phenotypes in fly and mice, together with the structural conservation of SWS/NTE, suggests that these proteins have a fundamental biological function in survival and integrity of the aging CNS.

In this study, we performed esterase assays to determine whether SWS shares the biochemical properties of NTE. We immunolocalized the fly protein to the endoplasmic reticulum, and we examined membrane lipid composition in the mutant. In addition, we performed transgenic rescue experiments to confirm that SWS and NTE are true orthologs as a means of investigating the conserved role of these proteins in maintaining neuronal survival.

\section{Materials and Methods}

Drosophila stocks. sws alleles have been described previously (Kretzschmar et al., 1997). Canton S was used as wild type-stock. The loco-GAL4 line was kindly provided by C. Klämbt (Universitat Muenster, Muenster, Germany), and the tracheal-GAL4 line was provided by A. Brand (University of Cambridge, Cambridge, UK). Actin-GAL4, glass multimer receptor (GMR)-GAL4, and elav-GAL4 were provided by the Bloomington Stock Center (Bloomington, IN). Stocks were maintained and raised under standard conditions.

sws-constructs. The Drosophila sws cDNA (GH08579) was obtained from the Berkeley Drosophila Genome Project (Berkeley, CA) and inserted in the P-element transformation vector pUAST, which was also used to obtain the murine sws transgene (Moser et al., 2000). The serine $^{985}$-to-aspartate mutation was created by substituting the AGC triplet by a GAC in a corresponding primer. A 357 bp PCR product containing this triplet was used to substitute this region in the wild-type cDNA.

Esterase assays. Frozen flies were homogenized (5\% w/w) in $50 \mathrm{~mm}$ Tris- $\mathrm{HCl}$ and $1 \mathrm{~mm} \mathrm{EDTA.} \mathrm{After} \mathrm{brief} \mathrm{centrifugation}(500 \times g ; 5 \mathrm{~min})$, the homogenates were assayed as described by Johnson (1977). NTE-like activity is operationally defined as that portion of phenyl valeratehydrolyzing activity that is resistant to paraoxon (40 $\mu \mathrm{M}$; a nonneuropathic OP) but sensitive to mipafox (50 $\mu$; a neuropathic OP). The homogenates were preincubated $\left(37^{\circ} \mathrm{C} ; 20 \mathrm{~min}\right)$ with paraoxon in the presence or absence of mipafox before substrate (phenyl valerate) was added, and the reaction was allowed to proceed for an additional $20 \mathrm{~min}$. Activity was determined as the amount of phenol liberated by this reaction and expressed as NTE-like activity (i.e., the difference in the activity measured in the presence and absence of mipafox) per milligram of homogenate protein. Homogenates were prepared from $\sim 200$ flies $(2-5$ $\mathrm{d}$ of age), and measurements were repeated at least twice, unless otherwise stated. Results are shown as mean values \pm SEM.

Tissue sections for light and electron microscopy. Fly heads were prepared for light and electron microscopy as described by Kretzschmar et al. (1997). For light microscopy, $2 \mu \mathrm{m}$ serial sections were cut and stained with $1 \%$ toluidine blue plus 1\% borax. Ultrathin Epon plastic sections were poststained with $2 \%$ uranyl acetate followed by Reynold's lead citrate (Reynolds, 1963) and stabilized for transmission electron micros- copy by carbon coating. Analysis of the specimens was done with a Zeiss (Oberkochen, Germany) EM10C/VR electron microscope at 40-80 kV. Glial cell material was identified clearly by its characteristically higher electron density (Saint Marie and Carlson, 1983a,b).

Immunohistochemistry. Paraffin sections were performed as described by Jäger and Fischbach (1989). The paraffin was removed with SafeClear (Fisher Scientific, Houston, TX), and the sections were rehydrated in graded ethanol. Sections were processed further as described by Buchner et al. (1989). An SWS fusion protein (QIAexpress system; Qiagen, Hilden, Germany) was used to raise antisera in rabbits and was applied in a 1:100 dilution in PBS (Buchner et al., 1989). An antibody against rat 78 $\mathrm{kDa}$ glucose-regulated protein Grp78 (Stressgen, Victoria, British Columbia, Canada) was used in a 1:1000 dilution to label the endoplasmic reticulum. Monoclonal antibodies against the neuronal protein embryonic lethal abnormal vision (ELAV) and the glial protein reversed polarity (REPO) were obtained from the Developmental Studies Hybridoma Bank (University of Iowa, Iowa City, IA). Supernatants were diluted 1:5 (REPO) and 1:100 (ELAV). Cyanine 2 (Cy2) and Cy3 secondary antibodies were obtained from Jackson ImmunoResearch (West Grove, PA).

Western blot. Western blots were performed as described by Tschape et al. (2002). Homogenates were prepared from fly heads from 3- to 6-d-old flies. The anti-sws antibody was used in a 1:500 dilution, and the protein was detected using the ECL system (Amersham Biosciences, Arlington Heights, IL). The detected SWS protein was $\sim 180 \mathrm{kDa}$ larger than the predicted size of $160 \mathrm{kDa}$, which could be a result of protein modifications.

Lipid and sterol measurements. Twenty milligrams of heads from 8- to 10-d-old flies were homogenized mechanically, and chloroform/methanol was extracted as described by Folch et al. (1957). Phospholipids were separated by two-dimensional thin-layer chromatography on silica gel 60 plates (Merck, Darmstadt, Germany) using chloroform/methanol/25\% $\mathrm{NH}_{3}$ (65:35:5; per volume) as the first solvent and chloroform/acetone/ methanol/acetic acid/water (50:20:10:10:5; per volume) as the second solvent. Phospholipids were visualized on TLC plates by staining with iodine vapor, scraped off, and quantified (Broekhuyse, 1968).

For the analysis of neutral lipid, extracts were applied to silica gel 60 plates with a sample applicator (Linomat IV; CAMAG, Basel, Switzerland), and chromatograms were developed in an ascending manner using light petroleum/diethyl ether/acetic acid (25:25:1; per volume) as the solvent for the first third of the total distance. Then the plates were dried briefly and further developed to two-thirds of the total distance by using light petroleum/diethyl ether (49:1; per volume). Quantitation of sterol and steryl ester was performed by densitometric scanning at $275 \mathrm{~nm}$ with ergosterol as a standard. Triacylglycerols (TAG) were visualized by postchromatographic staining using a chromatogram immersion device (CAMAG). Quantification of TAG was performed by densitometric scanning at $400 \mathrm{~nm}$ with triolein as a standard (NuCheck, Elysian, $\mathrm{MN}$ ). Results are shown as mean values \pm SEM from two to five independent measurements.

\section{Results}

Drosophila SWS is required autonomously in neurons and glia to rescue the sws mutant phenotype

To understand the cellular requirements of Drosophila SWS (DSWS) for brain integrity, we studied its function in different cell types within the adult brain. Previous in situ hybridization histochemical experiments on adult head sections had revealed the expression of sws mRNA in the entire brain cortex, which houses the neuronal cell bodies as well as cortex glial cells. In contrast, the neuropil, which contains only glial and tracheal cell bodies but no neuronal somata, showed no staining, indicating that this gene is not expressed in neuropil glia (Kretzschmar et al., 1997). To determine whether DSWS is required functionally in either neurons or glia, we expressed recombinant DSWS selectively in each cell type within sws flies. Surprisingly, the induction of neuronal expression of DSWS in mutant flies (using elavGAL4 as a neural-specific driver) suppressed only some aspects of 

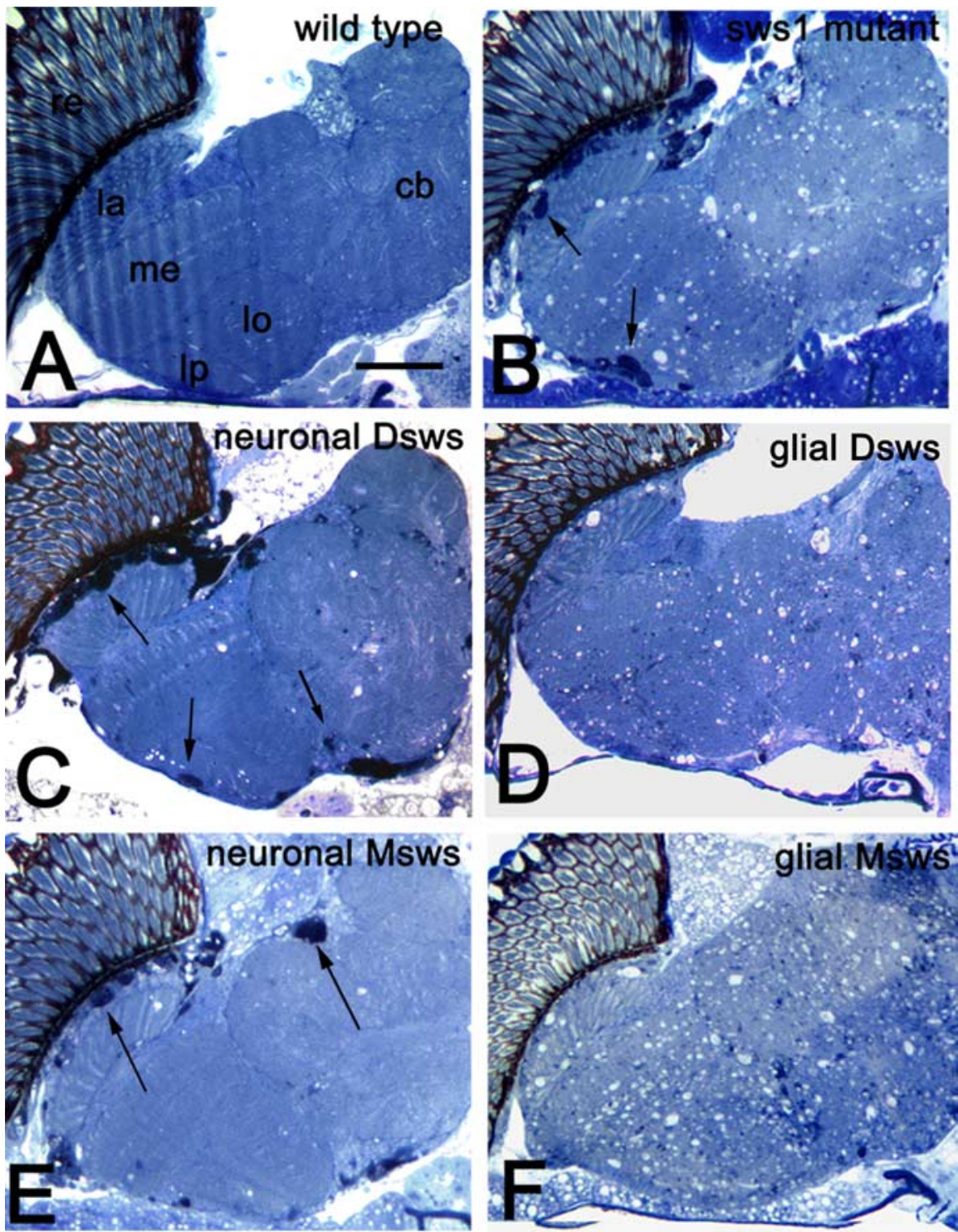

Figure 1. DSWS and MSWS can restore the wild-type function in glia and neurons. $\boldsymbol{A}$, Wild type. $\boldsymbol{B}$, An sws $^{1}$ fly shows the characteristic vacuolization in the neuropil and the formation of membranous glial bodies (arrows). C, Expressing the Drosophila gene in neurons almost completely inhibits the formation of vacuoles, whereas the glial phenotype still persists (arrows). D, Vice versa expression of DSWS in glia prevents the glial phenotype but not the neuronal vacuolization. The murine SWS can completely substitute the Drosophila gene and rescues the neuronal phenotype when expressed in neurons $(\boldsymbol{E})$ and the glial phenotype when expressed in glia $(\boldsymbol{F})$. All sections are semithin horizontal sections through the heads of 14-d-old flies. Neuropils of the optic lobes lamina (la), medulla (me), lobula (lo), and lobula plate (Ip); the retina (re); and the central brain (cb) are indicated. The corresponding cortex surrounds these fiber structures. Scale bar, $50 \mu \mathrm{m}$.

the degeneration, the vacuolization in the neuropil (Fig. $1 C$ ). In contrast, the glial phenotype, which consists of the formation of multilayered glial wrappings (Figs. $1 C, 2 \mathrm{~A}$, arrows) and subsequent glial cell death (Fig. 2D), persisted. Conversely, expression of DSWS in glia (using the pan-glial loco-GAL4 driver) rescued only the glial phenotype (Fig. $1 D$ ). The vacuolization, caused by degeneration of neurites (Fig. $2 B$ ), was still detectable. These results reveal a cell-autonomous requirement of DSWS in CNS neurons as well as in cortical glia. Expression in tracheal cells had no effect, nor could we rescue the sws phenotype by using a heatshock-inducible ubiquitous promoter construct (data not shown). The latter result suggests a continuous requirement of SWS in the nervous system that cannot be provided by periodic transient expression of the gene.

\section{Murine and Drosophila SWS are}

functionally conserved

Fly and vertebrate NTE/SWS proteins are conserved highly on both the sequence $(39 \%$ identical) and structural level (Moser et al., 2000). To determine whether the function of these proteins is similarly conserved, we asked whether murine SWS/NTE (MSWS) could restore the wild-type function of DSWS in sws mutant flies. We expressed MSWS in either glia or neurons in mutant flies, using the same promoter constructs described above. Notably, we found that the murine SWS/NTE gene was as effective as the fly sws gene in rescuing the mutant phenotype. As in the case of DSWS, we found that MSWS is required in glia and neurons in a cell-autonomous manner (Fig. $1 E, F$ ). These results confirm that the mouse protein (and therefore most likely also the human NTE protein, which is $96 \%$ identical to the MSWS) (Moser et al., 2000) is a true functional ortholog of fly SWS.

SWS is expressed in neurons and glia and is localized in the endoplasmic reticulum

To determine the expression pattern and intracellular localization of fly SWS, we produced antisera against DSWS. Immunohistochemistry on wild-type head sections from 1-d-old flies revealed a weak expression in most or all cells localized in the cortex (Fig. $3 A, B$ ). This is in agreement with the mRNA in situ hybridization data, which showed a similar pattern of DSWS mRNA expression throughout the cortex (Kretzschmar et al., 1997). By analogy to the dynamics of SWS/NTE expression in vertebrates, we also found that DSWS was preferentially expressed by specific subsets of neurons in older (12 d) animals (Fig. 3C, arrows).

The cortex region, in addition to housing neuronal cell bodies, also houses some glial cells. To determine whether these glial cells express DSWS (as suggested by the rescue experiments), we performed double stainings using antibodies against DSWS and the glia-specific REPO protein (Xiong et al., 1994). DSWS is expressed in the interface glia (Kretzschmar and Pflugfelder, 2002), which is localized at the border of the cortex and the neuropil (Fig. 3D) but could not be detected in neuropil glia in the protocerebrum (Fig. $3 E)$. In the optic system, especially in the lamina, the glia populations are more complex, and distinct types of glia have been described (Kretzschmar and Pflugfelder, 2002). In the lamina, expression was found in the fenestrated and satellite glia (Fig. 3E, white arrowheads) but not in the epithelial glia (Fig. 3F, black arrows). Another population that does not express detectable amounts of DSWS is the giant glia, localized at the optic chiasm (Fig. 3F, white arrow), whereas the medulla neuropil glia, local- 
ized at the medulla cortex-neuropil interface, revealed expression (Fig. 3F, black arrowheads).

DSWS protein was found in the cytoplasm (Fig. 3G) in a pattern that suggested localization to the ER. A similar localization to the ER was also observed after expression of green fluorescent proteintagged constructs of NTE in COS cells (Li et al., 2003) and by immunohistochemistry of endogenous NTE in cultured mouse hippocampal neurons (Akassoglou et al., 2004). To confirm this observation, we used an antibody recognizing the ER marker protein Grp78 (Fig. 3H). The colocalization of DSWS and Grp78 demonstrates that much of DSWS resides in the ER (Fig. 3I, arrow), with some DSWS found in vesicles not belonging to the ER (Fig. 3I, arrowheads).

\section{NTE-like activity is required for the physiological function of SWS} Mammalian NTE catalyzes the OPsensitive hydrolysis of the artificial substrate phenyl valerate, and Ser ${ }^{966}$ of NTE has been identified as its active site residue (Atkins and Glynn, 2000). The homology between DSWS and NTE is particularly marked (57\% identity) within the $\sim 500$ residue catalytic domain surrounding its active site, including conservation of a Ser residue at an equivalent position in DSWS $\left(\operatorname{Ser}^{985}\right)$. The $s w s^{1}$ mutation produces a truncated SWS polypeptide terminated at residue 375 , which thus lacks the esterase domain. We found readily detectable NTE-like activity in homogenates of wildtype flies $(56 \pm 20 \mathrm{nmol} / \mathrm{min}$ per milligram of protein; $n=6)$ but not in $s w s^{1}$ mutants $(4.7 \pm 3.6 \mathrm{nmol} / \mathrm{min}$ per milligram of protein; $n=5$ ) (Fig. $4 A$ ). Expression of DSWS in either neurons (using the elav-GAL4 driver) or glia (using the locoGal 4 driver) of $s w s^{1}$ mutants restored NTE-like activity (Fig. 4A). These results reveal that DSWS, like NTE, has an in vitro esterase activity.

In addition, expression of the murine ortholog in the mutant flies led to dramatic increases in esterase activity. An approximately sixfold increase was seen when MSWS was expressed in neurons, whereas a $\sim 60$-fold increase was seen when it was expressed in glial cells (Fig. 4A). Because it appears unlikely that the expression level of MSWS is 10 times higher than that of other tested constructs induced with the same promoter, these results suggest that, compared with DSWS, the murine protein is intrinsically more active in catalyzing hydrolysis of the artificial substrate phenyl valerate. As already noted, Ser ${ }^{985}$ of DSWS corresponds to the active site serine residue Ser ${ }^{966}$ of human NTE. To investigate whether this serine (and therefore esterase activity) is required for the in vivo function of DSWS, we exchanged Ser ${ }^{985}$ in
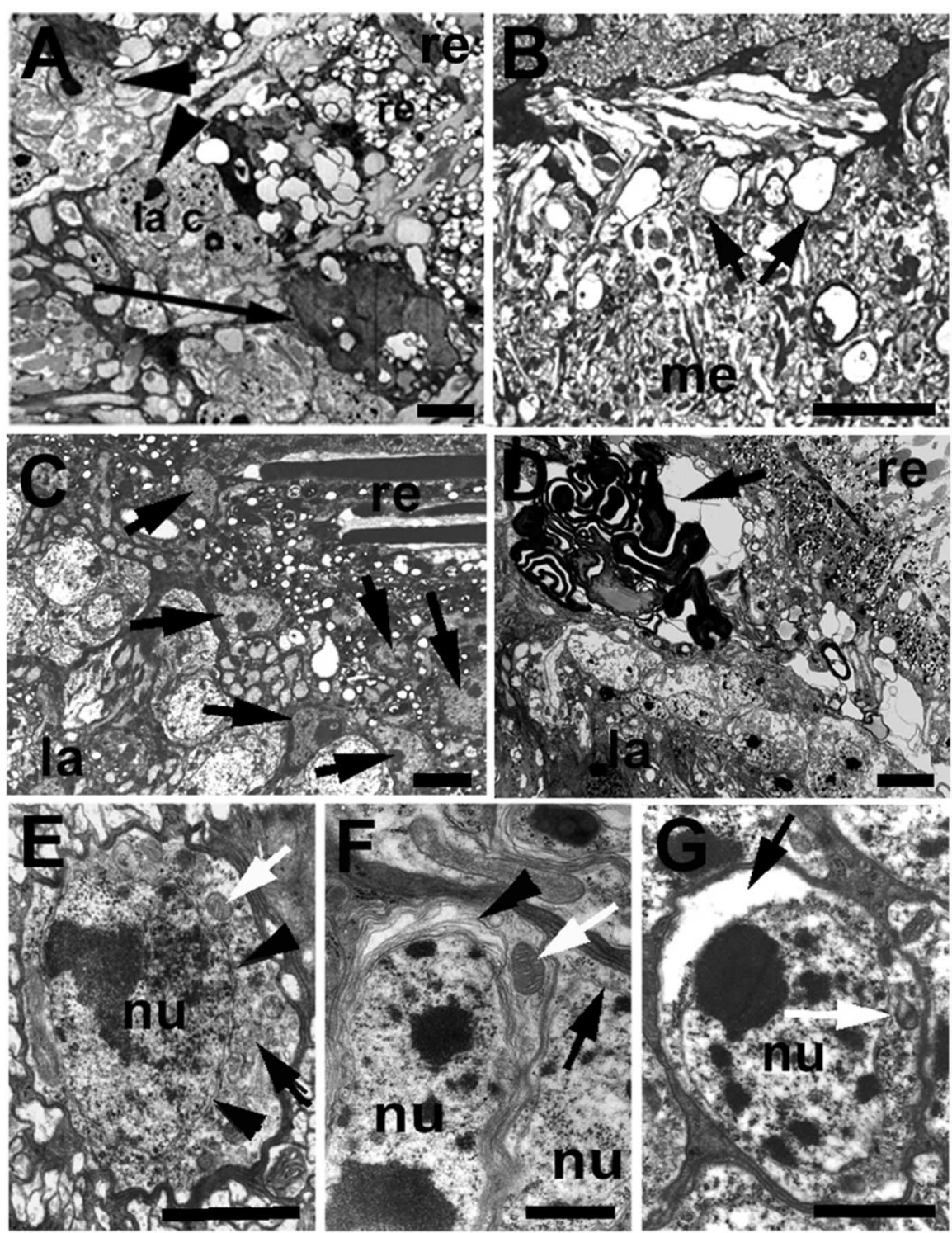

Figure 2. Electron microscopy reveals membrane defects in sws mutants. $A$, Ultrastructural analysis of a $14-\mathrm{d}-$ old sws $^{1}$ fly expressing DSWS in neurons reveals that the persisting glial phenotype consists of multilayered glial whorls (arrow). In contrast, the neurons in the lamina cortex (arrowheads) appear to be wild type. $\boldsymbol{B}$, Expressing DSWS in glia restores the glial phenotype; the vacuolization in the neuropil (arrows), attributable to degenerated axons and dendrites, however, is still apparent. C, In wild type, several glial cells (fenestrated and satellite glia; arrows) can be found in the lamina cortex. D, In a 20-d-old sws ${ }^{7}$ fly with neuronal expression of DSWS, the membranous glial structures (arrow) are the only remnants of glial cells, whereas intact glial cell bodies are completely absent. $\boldsymbol{E}$, A wild-type neuron shows an intact ER (arrowheads) and Golgi (black arrow) network, as well as mitochondria (white arrow). $\boldsymbol{F}$, A neuron in a 7-d-old sws ${ }^{7}$ mutant shows a number of disorganized membranes presumably originating from the ER (arrowhead), whereas the mitochondria are intact (white arrow). A neighboring neuron is still intact (black arrow). G, After 20 d of aging, an empty space (black arrow) has developed next to the nucleus, whereas mitochondria still appear to be normal (white arrow). re, Retina; la c, lamina cortex; la, lamina; me, medulla; nu, nucleus. Scale bars: $\boldsymbol{A}-\boldsymbol{D}, 2 \mu \mathrm{m} ; \boldsymbol{E}-\mathbf{G}, 1 \mu \mathrm{m}$.

DSWS for an aspartate. An immunoblot blot analysis was used to determine that the expression level of this construct was not significantly different from the wild-type construct (Fig. $4 C$ ), and immunohistochemistry on sections showed a similar distribution as for expression of the wild-type construct (Fig. 5D). Homogenates from lines expressing this mutant form either ubiquitously (data not shown) or specifically in neuronal cells did not show any enzymatic activity (Fig. $4 A$ ), confirming the importance of this residue for the esterase activity. A similar effect was observed when the mutant construct was expressed in glial cells (data not shown). More importantly, this construct was not able 

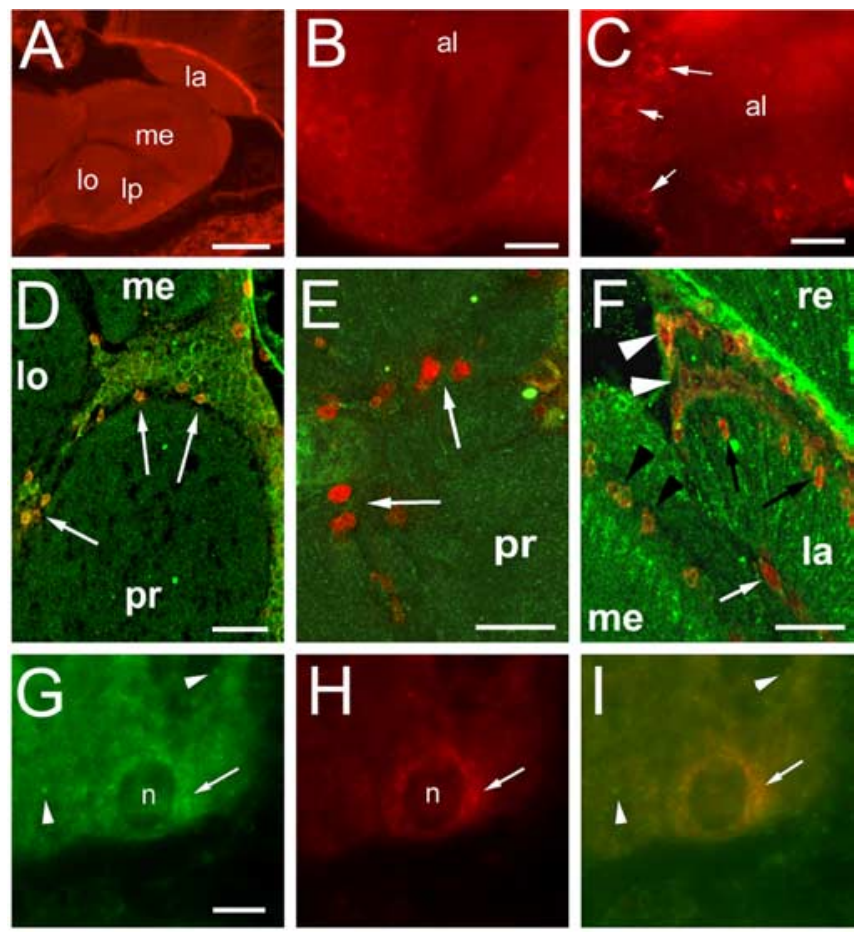

Figure 3. Expression of the DSWS protein. $A, B$, In a 1-d-old wild type, DSWS is expressed in most or all neurons. $\boldsymbol{C}$, In 12 -d-old flies, the pattern becomes more restricted, with some neurons (arrows) containing higher levels of protein (compare with $\boldsymbol{B})$. $\boldsymbol{D}-\boldsymbol{F}$, Double staining with anti-DSWS (green) and the glia-specific protein REPO (red) reveals expression in interface glia at the border of the neuropil and cortex ( $\boldsymbol{D}$, arrows) but not in the neuropil glia ( $\boldsymbol{E}$, arrows) of the protocerebrum. $\boldsymbol{F}$, In the optic system, we could not detect DSWS in the epithelial glia of the lamina (black arrows) and the giant glia at the optic chiasm (white arrow). In contrast, DSWS is expressed in the fenestrated and satellite glia in the lamina cortex (white arrowheads) and the medulla glia (black arrowheads) at the cortex-neuropil interface. $\boldsymbol{G}$, The DSWS protein is found in the cytoplasm of neurons and appears to be localized in vesicles (shown in the cortex of the central brain). The endoplasmic reticulum marker $\operatorname{Grp} 78(\boldsymbol{H})$ colocalizes with DSWS $(\boldsymbol{I})$, suggesting that most of the DSWS protein localizes to the endoplasmic reticulum (arrow) and only some DSWS in other vesicular structures (arrowheads). Horizontal cryosections from heads were used. la, Lamina; me, medulla; lo, lobula; Ip, Iobula plate; al, antennal lobe; pr, protocerebrum; n, nucleus; re, retina. Scale bars: $\boldsymbol{A}, 50 \mu \mathrm{m} ; \boldsymbol{B}, \boldsymbol{C}, 10 \mu \mathrm{m} ; \boldsymbol{D}-\boldsymbol{F}, 20 \mu \mathrm{m} ; \boldsymbol{G}, 1 \mu \mathrm{m}$.

to rescue the mutant phenotypes when expressed under the same conditions as the wild-type sws construct (data not shown). These results indicate that the catalytic activity of DSWS is required for its biological function.

\section{Overexpression and ectopic expression of catalytically active DSWS induces degeneration in wild-type Drosophila}

To determine whether elevated levels of DSWS have adverse effects on cell survival, we induced DSWS expression in wild-type flies. Homogenates from flies expressing DSWS under the control of the neuronal elav-GAL4 driver showed a twofold increase in NTE-like activity (Fig. $4 \mathrm{~B}$ ), and immunohistochemical staining for DSWS revealed a substantial amount of protein accumulating in vesicles within neuronal somata (Fig. $5 B, C$ ). However, histological examination of the brains of these animals revealed no neurodegenerative phenotypes. In contrast, induction of additional DSWS in glial cells (using the loco-GAL4 driver) caused a 12-fold increase in NTE-like activity (Fig. $4 \mathrm{~B}$ ) and strong expression levels in CNS glial cells (Fig. 5E,F). Histological sections of the brains of these flies revealed an age-dependent degeneration of CNS glia, particularly in the epithelial glia of the lamina and the giant glia within the first optic chiasm (Kretzschmar and Pflugfelder, 2002). Sections from newly eclosed flies revealed that the initial development of these glial cells appeared to be normal (data not shown). However, in 14-d-old flies, the remnants of these glial cells were filled completely with vacuoles (Fig. $6 \mathrm{~B}$ ) and numerous membranous cellular bodies (Fig. $6 B, C$ ). First signs of this degenerative process could already be found in younger flies (5-7 d) in the presence of small vesicles (Fig. 6D, arrowheads) and membranous bodies inside glial cells. Considering the much higher increase in esterase activity seen in these animals despite the fact that only $\sim 25 \%$ of brain cells in Drosophila are glia (Pfrieger and Barres, 1995), these results indicate that the level of DSWS overexpression in glia induced by the loco-GAL4 driver was much greater than that in neurons induced by the elav-GAL4 driver. This enhanced level of expression most likely explains the severe degeneration of glia seen in these animals, although enhanced sensitivity of glial cells to SWS activity might also contribute to this effect.

Endogenous SWS is not detected in the eye, and the sws mutant has no eye phenotype (Kretzschmar et al., 1997). To study adverse effects of ectopic SWS in the eye, we expressed DSWS with the GMR-GAL4 promoter construct (Fig. 7A). Induction of DSWS in the eyes of wild-type flies caused a twofold increase in esterase activity (Fig. $4 B$ ) and progressive degeneration of photoreceptors (Fig. $7 C$ ). Together, these results reveal a requirement for the controlled spatial and quantitative expression of SWS.

To investigate whether the catalytic function of DSWS underlies the deleterious effects caused by overexpression and misexpression of this gene, we used the active site mutation (Ser ${ }^{985}$ Asp ${ }^{985}$ ) to test its effects when expressed in an eye-specific manner. This catalytically inactive construct did not induce any of the phenotypes seen when wild-type DSWS was expressed (data not shown), strongly suggesting that the esterase activity of DSWS is required for the induction of neurodegenerative effects.

\section{SWS activity regulates lipid composition}

Recently, it has been shown that NTE can degrade ER-associated PtdCho in cultured mammalian cell lines (Zaccheo et al., 2004). We therefore tested whether PtdCho levels were altered in the sws mutant. As shown in Figure $8 \mathrm{~A}$, we found that PtdCho levels in 8to 10 -d-old $s w s^{1}$ mutant heads were increased to $122 \%$ of wildtype control levels, whereas other membrane lipids were not significantly affected (e.g., phosphatidyl ethanolamine) (Fig. 8C). In addition, we found that both TAG and sterol ester levels were markedly reduced (52 and $43 \%$ of wild-type controls, respectively) (Fig. $8 B, D$ ). To confirm an influence of SWS on lipid composition, we also examined lipid levels in homogenates from flies overexpressing SWS in neurons. As already noted, these animals showed a twofold increase in NTE-like enzyme activity (Fig. $4 B$ ) but did not exhibit a neurodegenerative phenotype. In contrast to the effects seen in sws mutants, PtdCho was reduced to $85 \%$ of wild-type levels, whereas TAG was increased to $197 \%$ (Fig. 8A,B). A definitive assay of sterol ester levels in these flies could not be obtained as a result of the occurrence of additional, unusual peaks in the measurements. Nevertheless, these results support the conclusion that like mammalian NTE, SWS participates in the regulation of normal lipid composition in the CNS.

\section{Discussion}

The Drosophila sws mutant is characterized by progressive degeneration of the adult nervous system, associated with apoptotic cell death and the formation of spongiform lesions in the CNS. NTE, the mammalian ortholog of fly SWS, is also a molecular target of organophosphates. Organophosphate poisoning is a significant 
public health threat, contributing to three million acute severe cases of pesticide poisonings and 20,000 unintentional deaths each year (as estimated by the World Health Organization). Although NTE has been studied for over 20 years, its biological role is still unclear. The primary sequence of the Drosophila SWS protein is similar to mammalian NTE, and we have now confirmed the functional conservation of these proteins, as well. Expression of the murine gene in sws mutant flies can rescue the neurodegeneration phenotype induced by this mutation, as does expression of the fly gene (Figs. 1, 2).

NTE mRNA (Moser et al., 2000) and protein (Glynn et al., 1998) are present in most or all vertebrate neurons in juvenile stages, with a more restricted pattern of expression in older animals (Moser et al., 2000). In addition, vertebrate SWS/NTE is present in several non-neural tissues (Johnson, 1982; Moser et al., 2000), although within the mouse nervous system, NTE immunostaining appears to be absent from glia (Glynn et al., 1998). Moreover, recent studies have revealed a subcellular localization of NTE to the endoplasmic reticulum ( $\mathrm{Li}$ et al., 2003; Akassoglou et al., 2004). Similar to vertebrates, we have shown that he Drosophila SWS protein can be found in most or all neurons and primarily colocalizes with an ER protein. The observed ultrastructural defects seen in sws mutants, which are attributable to the disruptions in the ER, support a vital function of SWS in the maintenance of this organelle.

Our rescue experiments confirmed that SWS/NTE expression in neurons is essential for their survival. Surprisingly, however, we found that glial expression of this protein is also necessary for the integrity of the adult brain. The results of our studies using cell type-specific induction of SWS revealed that the mutant phenotype involves two independent aspects: axonal degeneration (visible by the spongiform lesions in the neuropil) and subsequent neuronal cell death were both ameliorated by neuronal-specific expression of SWS. In contrast, the glial phenotype, consisting of hyperwrapping and the formation of large membranous bodies, was rescued only by SWS/NTE expression in glia. These results show a cellautonomous requirement of SWS in both cell types in the adult brain of Drosophila. SWS is also expressed in lower amounts during development; however, we did not detect any obvious defects in glial or neuronal differentiation in earlier stages. The expression of SWS in specific glial subpopulations as well as neurons was confirmed by immunohistochemical staining of brain sections. The previous failure to observe vertebrate SWS/NTE in glia of the mammalian brain may either have been a result of insufficient sensitivity of the detection procedure used in previous studies or may be the result of authentic differences in species-specific expression patterns.

These results suggest that only adult or aged neurons are crit-
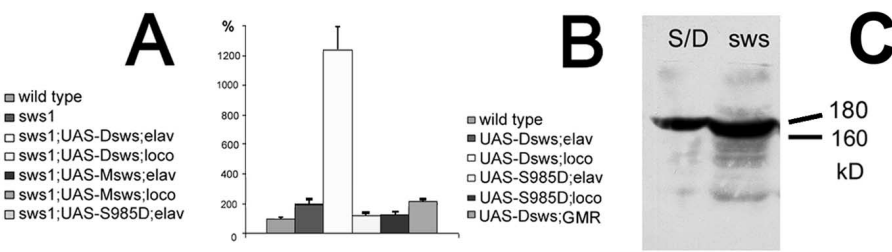

Figure 4. NTE-like esterase activity in fly homogenates. $\boldsymbol{A}$, Expression of fly or murine SWS in glia (loco-GAL4) or neurons (elav-GAL4) restores esterase activity in mutant sws $^{1}$ flies; in contrast, expression of the active site serine mutant form of DSWS values are expressed relative to wild type (100\%). *Because this genotype was obtained in low numbers, measurement was done only once. Error bars represent SEMS. C, Western blot analysis of SWS expression reveals that the loss of activity of the active site
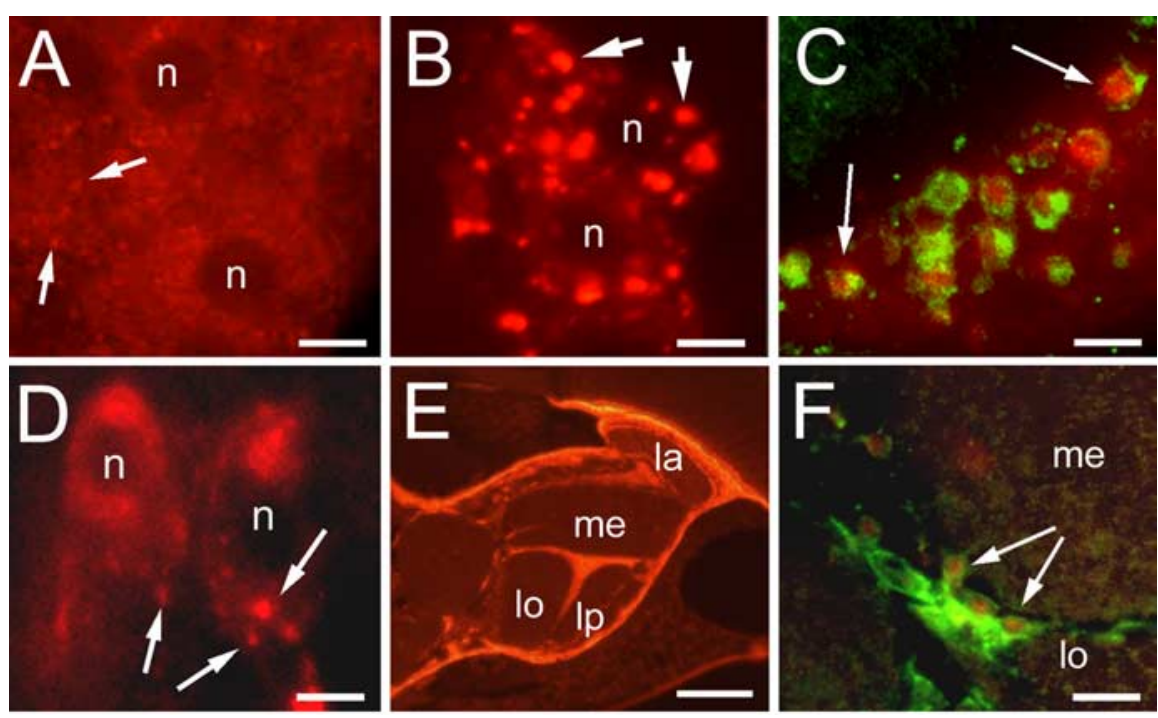

Figure 5. Overexpression of DSWS.A, Detection of the endogenous DSWS protein on wild-type head sections using anti-DSWS. $B, C$, Immunohistochemistry on head sections from an elav-GAL4, UAS-DSWS fly. Increased amounts and vesicular localization of DSWS (arrows) are prominent after neuronal overexpression. C, Double stainings with the neuronal specific anti-elav antibody serine mutant form of DSWS (S985D) in neurons (using elav-GAL4) also reveals localization of the mutant protein to vesicles. $\boldsymbol{E}, \boldsymbol{F}$ and the glia-specific anti-REPO antibody (red) confirm the localization in glia. la, Lamina; me, medulla; Io, Iobula; Ip, Iobula plate; al, antennal lobes; n, nucleus. Scale bars: $\boldsymbol{A}, \boldsymbol{B}, \boldsymbol{D}, 1 \mu \mathrm{m} ; \boldsymbol{C}, 2 \mu \mathrm{m} ; \boldsymbol{E}, 50 \mu \mathrm{m} ; \boldsymbol{F}, 3 \mu \mathrm{m}$.

ically dependent on SWS activity for their viability. Interestingly, toxicology studies in vertebrates have shown that older animals are substantially more sensitive to organophosphate-induced delayed neuropathy (Johnson and Barnes, 1970; Moretto et al., 1991). Alternatively, it is possible that defects resulting from aberrant SWS/NTE function accumulate only gradually and therefore do not manifest at other developmental stages.

Our studies also demonstrate that the functional conservation between fly and vertebrate SWS/NTE extends to their catalytic activities, because fly SWS exhibits an esterase activity resembling that of mammalian NTE, as measured by OP-sensitive hydrolysis of phenyl valerate. From a structural point of view, $\mathrm{Ser}^{985}$ in fly SWS aligns precisely with Ser ${ }^{966}$ of vertebrate NTE, which has been implicated as the active site residue. The failure of the point mutation in Ser ${ }^{985}$ to substitute wild-type SWS confirms the importance of the active site in vivo. More importantly, we found that the levels of PtdCho in extracts of fly heads were inversely related to the level of DSWS expression (Fig. 8). These findings are consistent with recent observations that NTE in both yeast and cultured mammalian cell lines degrades ER-associated Ptd- 

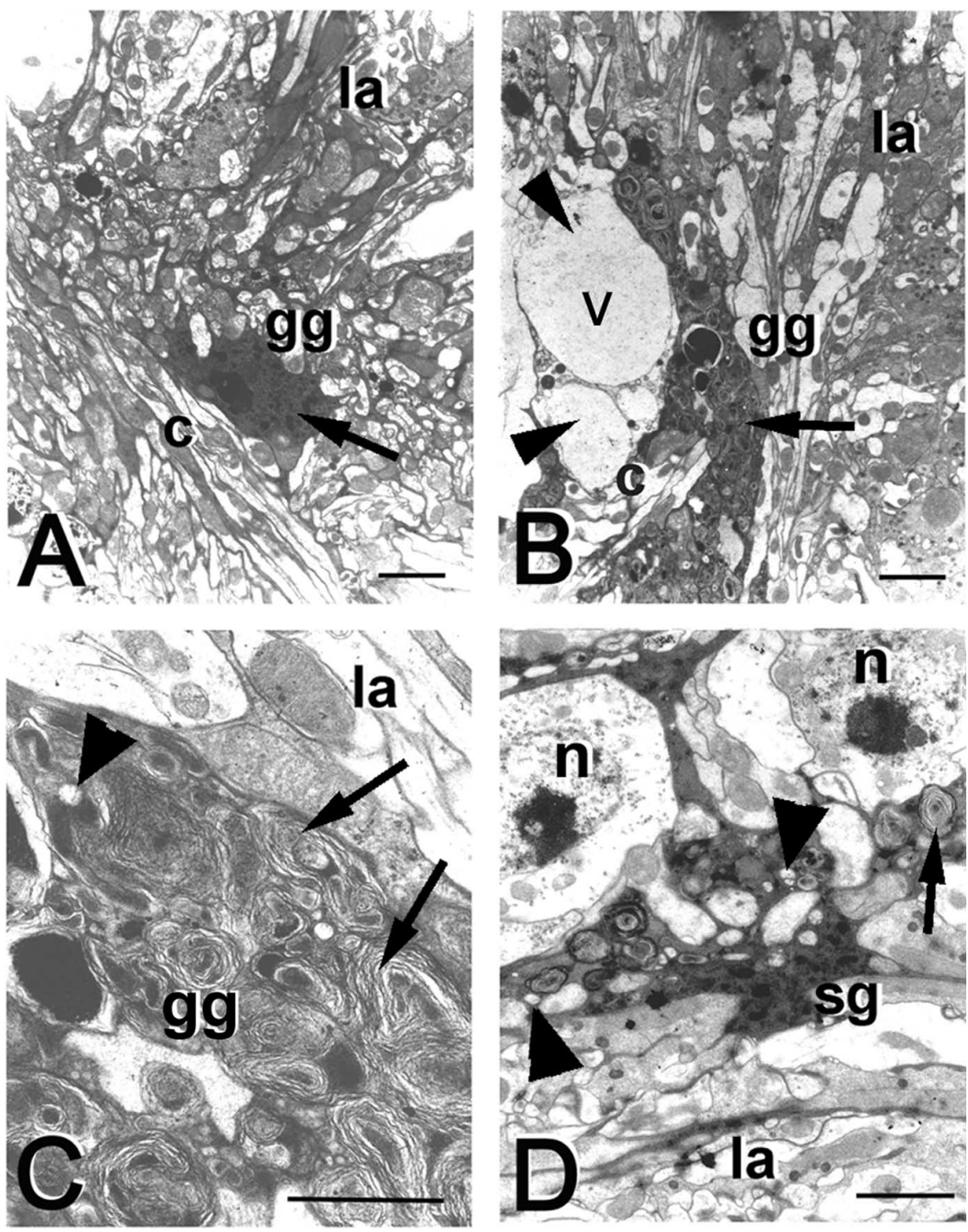

Figure 6. Glial cell death induced by overexpression of DSWS. $A$, Electron microscopic picture of a wild-type electron-dense giant glial cell ( $g$ g) at the first optic chiasm connecting lamina and medulla (c). B, A 14-d-old fly expressing additional DSWS in glia using the loco-GAL4 promoter construct. The giant glial cell is filled with membranous cellular bodies (MCBs) (arrow) and starts to go into lysis, which is visible by the formation of large vacuoles (v). $C$, Magnification from $\boldsymbol{B}$ showing the MCBs. $\boldsymbol{D}$, In a 7-d-old fly overexpressing DSWS, the MCBs are still small, and some vacuoles have formed (arrowheads). The glial cell shown is a satellite glial cell (sg) of the lamina (la). $n$, Nucleus. Scale bars: $A, B, 2 \mu \mathrm{m} ; C, D, 1 \mu \mathrm{m}$.
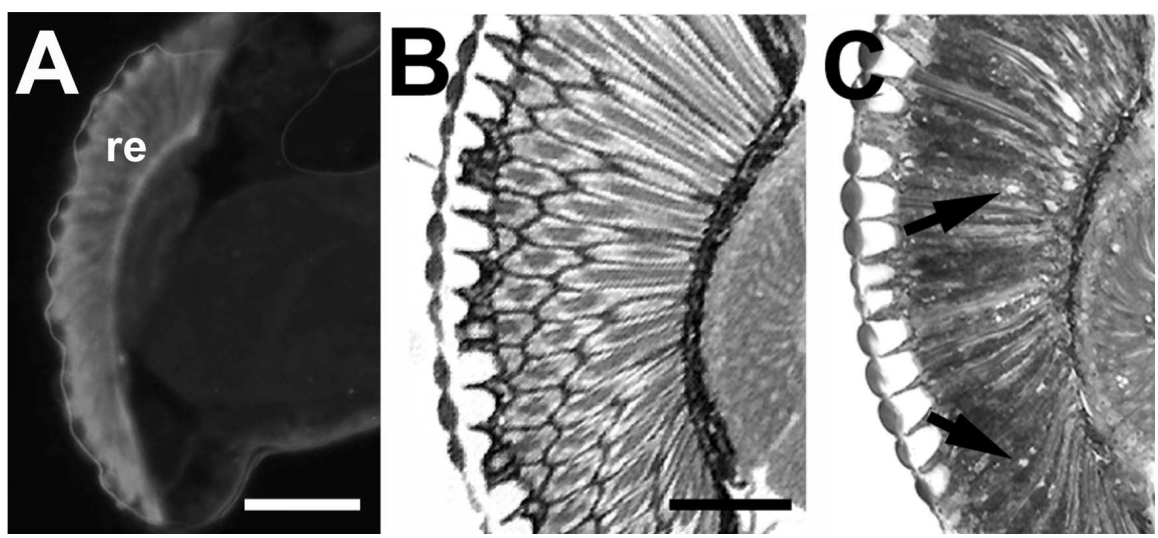

Figure 7. Ectopic expression of SWS in the eye. $\boldsymbol{A}$, Ectopic expression using the GMR-GAL4 promoter construct results in expression in photoreceptors. Immunohistochemistry on head sections using anti-DSWS is shown. $\boldsymbol{B}, \mathrm{A}$ semithin horizontal section from a wild-type eye. C, A-7-d old fly that expresses DSWS shows degeneration of photoreceptors visible by the disorganized structure and the formation of vacuoles (arrows). re, Retina. Scale bars: $\boldsymbol{A}, 100 \mu \mathrm{m} ; \boldsymbol{B}, 50 \mu \mathrm{m}$.
Cho (Zaccheo et al., 2004). We also found that triacylglycerol levels in the fly brain changed in reciprocal manner compared with PtdCho levels. This result is consistent with the dynamics of lipid synthesis in mammalian cells, in which diacylglycerol has been shown to be the immediate precursor for either PtdCho or triacylglycerol. Thus, when PtdCho synthesis is partially inhibited, triacylglycerol levels rise, whereas when PtdCho synthesis is increased, triacylglycerol falls (Allan, 2000; Jackowski et al., 2000).

In addition, we found a severe reduction in sterol ester levels in the sws mutant. Cholesterol ester can be synthesized by transfer of an acyl group from PtdCho to cholesterol by lecithin acyltransferase (LCAT), a serum enzyme that acts on the surface of high-density lipoproteins (Jonas, 2000). Although LCAT is primarily synthesized in the liver, smaller amounts can be found in the brain, and cultured glial cells secrete significant amounts into the surrounding culture medium (Collet et al., 1999). However, the role of LCAT in the brain is not yet understood. SWS/NTE shows no significant sequence homology to LCAT, and an LCAT ortholog can be found in the Drosophila genome (http:// flybase.bio.indiana.edu). Therefore, we assume that SWS/NTE does not act directly as an LCAT-like enzyme but might influence brain LCAT via an alternative pathway. Although additional studies are required to determine the role of SWS/ NTE in cholesterol ester production, our biochemical results support the conclusion that SWS as well as NTE play a critical function in the regulation of membrane lipid composition in vivo.

If SWS/NTE does play a central role in the turnover of phospholipids associated with the ER membrane, then overexpression of this protein might be expected to cause deleterious changes in membrane composition and lead to aberrant membrane breakdown, particularly in the ER. This indeed appeared to be the consequence of overexpressing catalytically active DSWS in glia, an effect that was not seen when we overexpressed the serine-toaspartate mutant form (excluding the possibility that the toxicity was simply attributable to high levels of SWS expression). Conversely, a lack of DSWS/NTE activity would allow the abnormal accumulation of ER membrane phospholipid, providing a rationale for the defects seen in ER membranes of aged sws mutant animals. In this aspect, the Drosophila mutant appears to be different from yeast lacking the NTE homolog YML059c, which do not show 

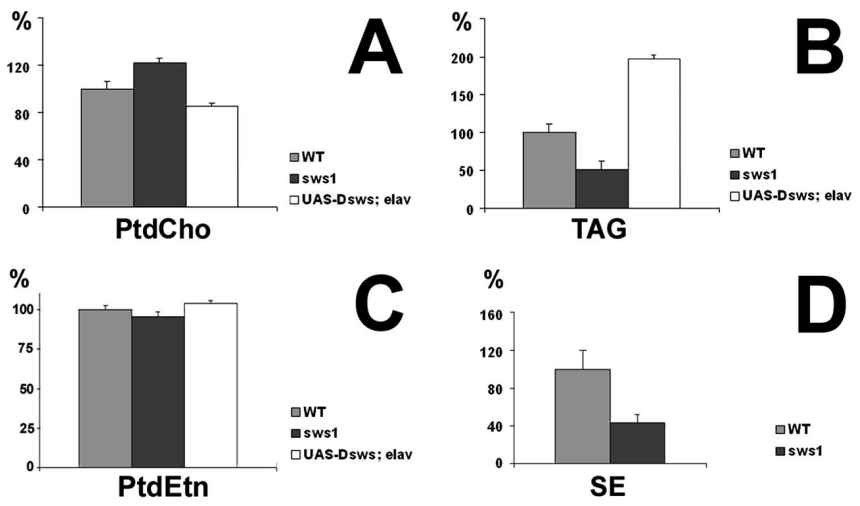

Figure 8. SWS affects the lipid content. Head homogenates from sws ${ }^{7}$ mutant flies and flies overexpressing DSWS in neurons were compared with wild-type (WT) controls. A, PtdCho levels are increased in the mutant and decreased in flies expressing additional SWS. $\boldsymbol{B}$, The opposite effect is seen for the levels of TAG. C, The level of PtdEtn is not significantly changed. D, Also, the level of sterol ester is reduced in the sws $^{7}$ mutant. All values are expressed relative to wild type (100\%). SEMs are indicated.

increased levels of PtdCho (Zaccheo et al., 2004). This result suggests that in contrast to yeast, which control PtdCho levels by downregulating the rate of PtdCho synthesis, metazoan cells may require the activity of SWS/NTE-related enzymes to maintain PtdCho homeostasis. PtdCho is a major component of most membranes, and disturbances in PtdCho metabolism have been associated with growth arrest and apoptotic cell death (Cui and Houweling, 2002; Wright et al., 2004). PtdCho depletion also induces expression of the ER-stress-related protein GADD153 (growth arrest and DNA-damage-inducible protein), which has been shown to have proapoptotic activity (van der Sanden et al., 2003). Similarly, PtdCho-specific phospholipase C has been implicated in triggering apoptosis in cells deprived of survival factors (Du et al., 2003). In addition, the enzymatic breakdown of phospholipids plays an important role in receptor-mediated signal transduction and cell communication (Divecha and Irvine, 1995). Breakdown of PtdCho has been observed in many acute and chronic neurodegenerative disorders, although it is not clear whether this event is a cause or a consequence of these disorders (Klein, 2000). Whereas there is ample evidence for the involvement of PtdCho breakdown in neurodegeneration, much less is known about the potential adverse effects of abnormally elevated PtdCho levels. Experiments in yeast suggest that excess PtdCho is intrinsically toxic to Golgi secretory function (Xie et al., 2001). It has also been reported that elevated PtdCho levels can be found in specific brain regions of Alzheimer's patients (Soderberg et al., 1992) and that PtdCho synthesis is increased in a dose-specific manner by amyloid $\beta$, the major component of the plaques found in Alzheimer's patients (Koudinova et al., 2000). PtdCho and related choline compounds have a neuronal-specific function, in that they have been shown to promote the synthesis and transmission of neurotransmitters (McDaniel et al., 2003). Thus, in addition to defects associated with membrane integrity, accumulation of excess PtdCho in neuronal axons and dendrites may also result in the misregulation of transmitter synthesis and release. Such an effect might further contribute to the degeneration of neurites and subsequent neuronal death, an effect that is compatible with the progressive appearance of vacuoles in the neuropil of sws mutant flies. The sws mutant thus provides an experimental model for functional studies of the role of SWS/NTE in maintaining neural integrity and can be used in future investigations to decipher the role of this conserved enzyme with respect to the mechanisms of OP-induced delayed neuropathy.

\section{References}

Akassoglou K, Malester B, Xu J, Tessarollo L, Rosenbluth J, Chao MV (2004) Brain-specific deletion of neuropathy target esterase/swisscheese results in neurodegeneration. Proc Natl Acad Sci USA 101:5075-5080.

Allan D (2000) Lipid metabolic changes caused by short-chain ceramides and the connection with apoptosis. Biochem J 345:603-610.

Atkins J, Glynn P (2000) Membrane association of and critical residues in the catalytic domain of human neuropathy target esterase. J Biol Chem 275:24477-24483.

Broekhuyse RM (1968) Phospholipids in tissues of the eye. Isolation, characterization and quantitative analysis by two-dimensional thin-layer chromatography of diacyl and vinyl-ether phospholipids. Biochem Biophys Acta 260: 449-459.

Buchner S, Buchner E, Hofbauer A (1989) Immunocytochemistry of the brain. In: Drosophila: a laboratory manual (Ashburner M, ed), pp 271273. Cold Spring Harbor, NY: Cold Spring Harbor.

Collet X, Francone O, Besnard F, Fielding CJ (1999) Secretion of lecithin: cholesterol acyltransferase by brain neuroglial cell lines. Biochem Biophys Res Commun 258:73-76.

Cui Z, Houweling M (2002) Phosphatidylcholine and cell death. Biochim Biophys Acta 1585:87-96.

Divecha N, Irvine RF (1995) Phospholipid signaling. Cell 80:269-278.

Du C, Zhao Q, Araki S, Zhang S, Miao J (2003) Apoptosis mediated by phosphatidylcholine-specific phospholipase $\mathrm{C}$ is associated with cAMP, p53 level, and cell-cycle distribution in vascular endothelial cells. Endothelium 10:141-147.

Dyer KR, el-Fawal HA, Ehrich MF (1991) Comparison of organophosphate-induced delayed neuropathy between branches of the tibial nerve and the biventer cervicis nerve in chickens. Neurotoxicology 12:687-695.

Dyer KR, Jortner BS, Shell LG, Ehrich M (1992) Comparative doseresponse studies of organophosphorus ester-induced delayed neuropathy in rats and hens administered mipafox. Neurotoxicology 13:745-755.

Folch J, Lees M, Sloane-Stanley GH (1957) A simple method for the isolation and purification of total lipids from animal tissues. J Biol Chem 226:497-509.

Glynn P (2000) Neural development and neurodegeneration: two faces of neuropathy target esterase. Prog Neurobiol 61:61-74.

Glynn P, Holton JL, Nolan CC, Read DJ, Brown L, Hubbard A, Cavanagh JB (1998) Neuropathy target esterase: immunolocalization to neuronal cell bodies and axons. Neuroscience 83:295-302.

Jackowski S, Wang J, Baburina I (2000) Activity of the phosphatidylcholine biosynthetic pathway modulates the distribution of fatty acids into glycerolipids in proliferating cells. Biochim Biophys Acta 1483:301-315.

Jäger RJ, Fischbach KF (1989) Mass histology of adult heads. In: Drosophila: a laboratory manual (Ashburner M, ed), pp 254-259. Cold Spring Harbor, NY: Cold Spring Harbor.

Johnson MK (1977) Improved assay of neurotoxic esterase for screening organophosphates for delayed neurotoxicity potential. Arch Toxicol 37:113-115.

Johnson MK (1982) The target for initiation of delayed neurotoxicity by organophosphorus esters: biochemical studies and toxicological applications: In: Reviews in biochemical toxicology, Vol 4 (Hodgson E, Bend JR, Philpot RM, eds), pp 141-212. New York: Elsevier.

Johnson MK (1990) Organophosphates and delayed neuropathy-is NTE alive and well? Toxicol Appl Pharmacol 102:385-399.

Johnson MK, Barnes JM (1970) Age and the sensitivity of chicks to the delayed neurotoxic effects of some organophosphorus compounds. Biochem Pharmacol 19:3045-3047.

Jonas A (2000) Lecithin cholesterol acyltransferase. Biochim Biophys Acta 1529:245-256.

Klein J (2000) Membrane breakdown in acute and chronic neurodegeneration: focus on choline-containing phospholipids. J Neural Transm 107:1027-1063.

Koudinova NV, Koudinov AR, Yavin E (2000) Alzheimer's Abeta1-40 peptide modulates lipid synthesis in neuronal cultures and intact rat fetal brain under normoxic and oxidative stress conditions. Neurochem Res 25:653-660.

Kretzschmar D, Pflugfelder GO (2002) Glia in development, function, and neurodegeneration of the adult insect brain. Brain Res Bull 57:121-131.

Kretzschmar D, Hasan G, Sharma S, Heisenberg M, Benzer S (1997) The 
swiss cheese mutant causes glial hyperwrapping and brain degeneration in Drosophila. J Neurosci 17:7425-7432.

Li Y, Dinsdale D, Glynn P (2003) Protein domains, catalytic activity and subcellular distribution of neuropathy target esterase in mammalian cells. J Biol Chem 278:8820-8825.

Lotti M (1992) The pathogenesis of organophosphate polyneuropathy. Crit Rev Toxicol 21:465-487.

Lush MJ, Li Y, Read DJ, Willis AC, Glynn P (1998) Neuropathy target esterase and a homologous Drosophila neurodegeneration-associated mutant protein contain a novel domain conserved from bacteria to man. Biochem J 332:1-4.

McDaniel MA, Maier SF, Einstein GO (2003) “Brain-specific" nutrients: a memory cure? Nutrition 19:957-975.

Moretto A (2000) Promoters and promotion of axonopathies. Toxicol Lett 112-113:17-21.

Moretto A, Capodicasa E, Peraica M, Lotti M (1991) Age sensitivity to organophosphate-induced delayed neuropathy: biochemical and toxicological studies in developing chicks. Biochem Pharmacol 41:1497-1504.

Moser M, Stempfl T, Li Y, Glynn P, Buttner R, Kretzschmar D (2000) Cloning and expression of the murine sws/NTE gene. Mech Dev 90:279-282.

Moser M, Li Y, Vaupel K, Kretzschmar D, Kluge R, Glynn P, Buettner R (2004) Placental failure and impaired vasculogenesis result in embryonic lethality for neuropathy target esterase-deficient mice. Mol Cell Biol 24:1667-1679.

Pfrieger FW, Barres BA (1995) What the fly's glia tell the fly's brain. Cell 83:671-674.

Reynolds ES (1963) The use of lead citrate at high $\mathrm{pH}$ as an electron-opaque stain in electron microscopy. J Cell Biol 17:208-212.

Saint Marie RL, Carlson SD (1983a) The fine structure of neuroglia in the lamina ganglionaris of the housefly, Musca domestica L. J Neurocytol $12: 213-241$

Saint Marie RL, Carlson SD（1983b） Glial membrane specializations and the compartmentalization of the lamina ganglionaris of the housefly compound eye. J Neurocytol 12:243-275.

Smith MI, Elove E, Frazier WH (1930) The pharmacological action of certain phenol esters with special reference to the etiology of the so-called Ginger paralysis. Public Health Rep 45:2509-2524.

Soderberg M, Edlund C, Alafuzoff I, Kristensson K, Dallner G (1992) Lipid composition in different regions of the brain in Alzheimer's disease/senile dementia of Alzheimer's type. J Neurochem 59:1646-1653.

Tschape JA, Hammerschmied C, Muhlig-Versen M, Athenstaedt K, Daum G, Kretzschmar D (2002) The neurodegeneration mutant lochrig interferes with cholesterol homeostasis and Appl processing. EMBO J 21:6367-6376.

van der Sanden MH, Houweling M, van Golde LM, Vaandrager AB (2003) Inhibition of phosphatidylcholine synthesis induces expression of the endoplasmic reticulum stress and apoptosis-related protein CCAAT/ enhancer-binding protein-homologous protein (CHOP/GADD153). Biochem J 369:643-650.

Winrow CJ, Hemming ML, Allen DM, Quistad GB, Casida JE, Barlow C (2003) Loss of neuropathy target esterase in mice links organophosphate exposure to hyperactivity. Nat Genet 33:477-485.

Wright MM, Howe AG, Zaremberg V (2004) Cell membranes and apoptosis: role of cardiolipin, phosphatidylcholine, and anticancer lipid analogues. Biochem Cell Biol 82:18-26.

Xie Z, Fang M, Bankaitis VA (2001) Evidence for an intrinsic toxicity of phosphatidylcholine to Sec14p-dependent protein transport from the yeast Golgi complex. Mol Biol Cell 12:1117-1129.

Xiong WC, Okano H, Patel NH, Blendy JA, Montell C (1994) repo encodes a glial-specific homeo domain protein required in the Drosophila nervous system. Genes Dev 8:981-994.

Zaccheo O, Dinsdale D, Meacock PA, Glynn P (2004) Neuropathy target esterase and its yeast homologue degrade phosphatidylcholine to glycerophosphocholine in living cells. J Biol Chem 279:24024-24033. 